\title{
Pressure dependence of the superconducting transition and electron correlations in $\mathrm{Na}_{x} \mathrm{CoO}_{2} \cdot 1.3 \mathrm{H}_{2} \mathrm{O}$
}

\author{
E. Kusano, ${ }^{1}$ S. Kawasaki, ${ }^{1}$ K. Matano, ${ }^{1}$ Guo-qing Zheng, ${ }^{1}$ R. L. Meng, ${ }^{2}$ J. Cmaidalka, ${ }^{2}$ and C. W. Chu ${ }^{2,3,4}$ \\ ${ }^{1}$ Department of Physics, Okayama University, Okayama 700-8530, Japan \\ ${ }^{2}$ Department of Physics and TCSAM, University of Houston, Houston, Texas 77204-5932, USA \\ ${ }^{3}$ Lawrence Berkeley National Laboratory, 1 Cyclotron Road, Berkeley, California 94720, USA \\ ${ }^{4}$ Hong Kong University of Science and Technology, Hong Kong, China
}

(Received 21 June 2007; revised manuscript received 8 August 2007; published 19 September 2007)

\begin{abstract}
We report $T_{c}$ and ${ }^{59} \mathrm{Co}$ nuclear quadrupole resonance measurements on the cobalt oxide superconductor $\mathrm{Na}_{x} \mathrm{CoO}_{2} \cdot 1.3 \mathrm{H}_{2} \mathrm{O}\left(T_{c}=4.8 \mathrm{~K}\right)$ under hydrostatic pressure $(P)$ up to $2.36 \mathrm{GPa}$. $T_{c}$ decreases with increasing pressure at an average rate of $-0.49 \pm 0.09 \mathrm{~K} / \mathrm{GPa}$. At low pressures $P \leqslant 0.49 \mathrm{GPa}$, the decrease of $T_{c}$ is accompanied by a weakening of the spin correlations at a finite wave vector and a reduction of the density of states (DOS) at the Fermi level. At high pressures above $1.93 \mathrm{GPa}$, however, the decrease of $T_{c}$ is mainly due to a reduction of the DOS. These results indicate that the electronic and magnetic state of Co is primarily responsible for the superconductivity. The spin-lattice relaxation rate $1 / T_{1}$ at $P=0.49 \mathrm{GPa}$ shows a $T^{3}$ variation below $T_{c}$ down to $T \sim 0.12 T_{c}$, which provides compelling evidence for the presence of line nodes in the superconducting gap function.
\end{abstract}

DOI: 10.1103/PhysRevB.76.100506

PACS number(s): 74.25.Nf, 74.62.Fj, 74.70.-b

The recently discovered superconductivity in cobalt oxide $\mathrm{Na}_{x} \mathrm{CoO}_{2} \cdot 1.3 \mathrm{H}_{2} \mathrm{O}$ has attracted considerable attention. ${ }^{1}$ This compound bears some similarities to the high- $T_{c}$ copper oxide superconductors, although Co forms a triangular lattice rather than a square lattice as in the cuprates. In an early study on a sample with large $\mathrm{Na}$ content, $x=0.31$ and $T_{c}=3.7 \mathrm{~K}$, it was found that the spin-lattice relaxation rate $1 / T_{1}$ shows a $T^{n}$ decrease with no coherence peak just below $T_{c}$, which suggests non- $s$-wave superconducting state. ${ }^{2}$ However, the crossover from $n=3$ to 1 at low temperatures, which was subsequently confirmed by other groups, has generated debate on whether there are nodes in the gap function. ${ }^{3,4}$ Later measurements on a low- $x$ sample with high $T_{c}(x$ $\left.=0.26, T_{c}=4.6 \mathrm{~K}\right)$ reveal that $1 / T_{1}$ follows a $T^{3}$ variation all way down to $T=T_{c} / 6$, indicating unambiguously that there exist line nodes in the gap function. ${ }^{5}$ It is also worth noting that the spin fluctuations at finite wave vector, most likely of antiferromagnetic (AF) origin, increase with decreasing $\mathrm{Na}$ content, and become strongest at $x \sim 0.26$ where $T_{c}$ is highest. $^{5}$

To investigate the spin-pairing symmetry, the Knight shift has been measured both in a powder sample ${ }^{6}$ and in a single crystal. ${ }^{7}$ It has been found that the shift decreases below $T_{c}$ along both the $a$-axis and $c$-axis directions. ${ }^{7}$ This indicates that the Cooper pairs are in the singlet form. Therefore, the experimental results strongly suggest that the superconductivity is of $d$-wave symmetry.

In this paper, we report nuclear quadrupole resonance (NQR) studies on $\mathrm{Na}_{x} \mathrm{CoO}_{2} \cdot 1.3 \mathrm{H}_{2} \mathrm{O}$ with the highest $T_{c}=4.8 \mathrm{~K}$ under pressure $(P)$, in order to get a hint as to the mechanism of the superconductivity. We investigate the correlation between $T_{c}$ and the electronic states. We have measured $T_{c}, \mathrm{NQR}$ frequency $\nu_{Q}$, and the spin-lattice relaxation rate $1 / T_{1}$ under pressures up to $2.36 \mathrm{GPa}$. We find that $T_{c}$ decreases with increasing pressure at an average rate of $-0.49 \pm 0.09 \mathrm{~K} / \mathrm{GPa}$. At low pressures $P \leqslant 0.49 \mathrm{GPa}$, the decrease of $T_{c}$ is due to a weakening of the spin correlations and a reduction of the density of states (DOS) at the Fermi level. At high pressures above $1.93 \mathrm{GPa}$, in contrast, the decrease of $T_{c}$ is due mainly to a reduction of the DOS. Our results indicate that the magnetic and electronic state of Co is primarily responsible for the superconductivity. Also, the spin-lattice relaxation rate $1 / T_{1}$ at $P=0.49 \mathrm{GPa}$ shows a $T^{3}$ variation below $T_{c}$ down to $T \sim 0.12 T_{c}$, as at ambient pressure,${ }^{5}$ which adds additional evidence for the presence of line nodes in the superconducting gap function.

The $\mathrm{Na}_{x} \mathrm{CoO}_{2} \cdot \mathrm{yH}_{2} \mathrm{O}$ powder ${ }^{8}$ was synthesized following Ref. 1. The Na content of this sample was not determined independently. Comparison of the $c$-axis length, $\nu_{Q}$, and $1 / T_{1}$ data with the previous group of samples ${ }^{5}$ shows that this sample has $x=0.26-0.28 . T_{c}$ was determined from the ac susceptibility and $1 / T_{1}$. ${ }^{59} \mathrm{Co}$ NQR measurements were carried out by using a phase-coherent spectrometer. The nuclear magnetization decay curve is excellently fitted to the theoretical formula 9

$$
\begin{aligned}
\frac{M_{0}-M(t)}{M_{0}}= & 0.095 \exp \left(-3 t / T_{1}\right)+0.095 \exp \left(-9.5 t / T_{1}\right) \\
& +0.819 \exp \left(-19 t / T_{1}\right),
\end{aligned}
$$

with a unique $T_{1}$ component. The hydrostatic pressure was applied by utilizing a NiCrAl-BeCu piston-cylinder-type cell, filled with Daphne 7373 oil as a pressure-transmitting medium. ${ }^{10}$ The value of pressure at low temperatures was determined from the $T_{c}$ value of $\mathrm{Sn}$ metal measured by a conventional four-terminal method. ${ }^{11}$ For $P$ other than $0.49 \mathrm{GPa}$, all measurements were done during the same experimental run at each pressure. For $P=0.49 \mathrm{GPa}$, measurements above $1.4 \mathrm{~K}$ were carried out during one experimental run; then the pressure cell was warmed up and attached to a ${ }^{3} \mathrm{He}$ refrigerator for data acquisition below $1.4 \mathrm{~K}$.

Figure 1 shows the ac susceptibility measured using the in situ NQR coil. $T_{c}$ was determined as the onset temperature of the diamagnetism, which is in agreement, within $\pm 0.1 \mathrm{~K}$, 


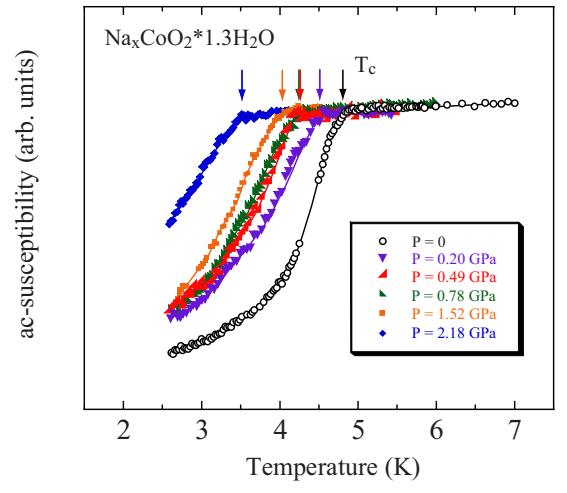

FIG. 1. (Color online) Typical examples of the ac susceptibility measured using the NQR coil. The arrows indicate $T_{c}$ at different pressures.

with the temperature at which $1 / T_{1}$ decreases (see below).

Figure 2 shows the $\pm 5 / 2 \leftrightarrow \pm 7 / 2$ NQR transition line at three typical pressures. For convenience, we call this resonance frequency $3 \nu_{Q}$; it increases with increasing pressure. It is noted that the width of the transition does not depend on pressure, indicating that the quality of the sample is unaffected by applying pressure.

Figure 3 shows the temperature dependence of the quantity $1 / T_{1} T$ at ambient and high pressures. The data for pressures of 1.27 and $1.93 \mathrm{GPa}$ are very similar to those at $0.49 \mathrm{GPa}$ and are not shown for the sake of clarity. Overall, the absolute value of $1 / T_{1} T$ decreases with increasing $P$. The extent of the progressive increase in $1 / T_{1} T$ upon lowering $T$ also changes (see later discussion). It has been noted that the non-Korringa behavior, namely, the increase of $1 / T_{1} T$ upon lowering $T$, is a signature of spin correlations. ${ }^{2,5}$ Since the Knight shift along both $a$ and $c$ axes decreases with decreasing $T$ and becomes a constant at $T_{c} \leqslant T \leqslant 100 \mathrm{~K}$, the spin correlations are not ferromagnetic in origin; ${ }^{12}$ the facts rather point to antiferromagnetic spin fluctuations.

In order to quantify the discussion, we analyze our data in the same way as for the ambient pressure data. In a general form, $1 / T_{1} T$ is written as

$$
1 / T_{1} T=\sum_{q} A_{q}^{2} \chi^{\prime \prime}(q, \omega) / \omega
$$

in the $\omega \rightarrow 0$ limit, where $A_{q}$ is the hyperfine coupling constant, and $\chi^{\prime \prime}(q, \omega)$ is the imaginary part of the $q$-dependent,

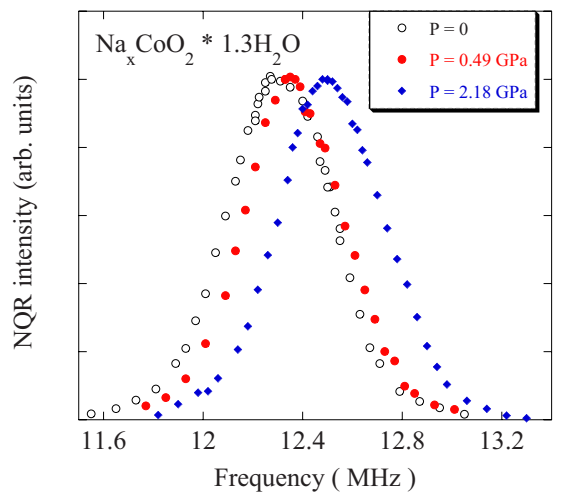

FIG. 2. (Color online) ${ }^{59} \mathrm{Co} \mathrm{NQR}$ spectra at the $\pm 5 / 2 \leftrightarrow \pm 7 / 2$ transition under three different pressures.

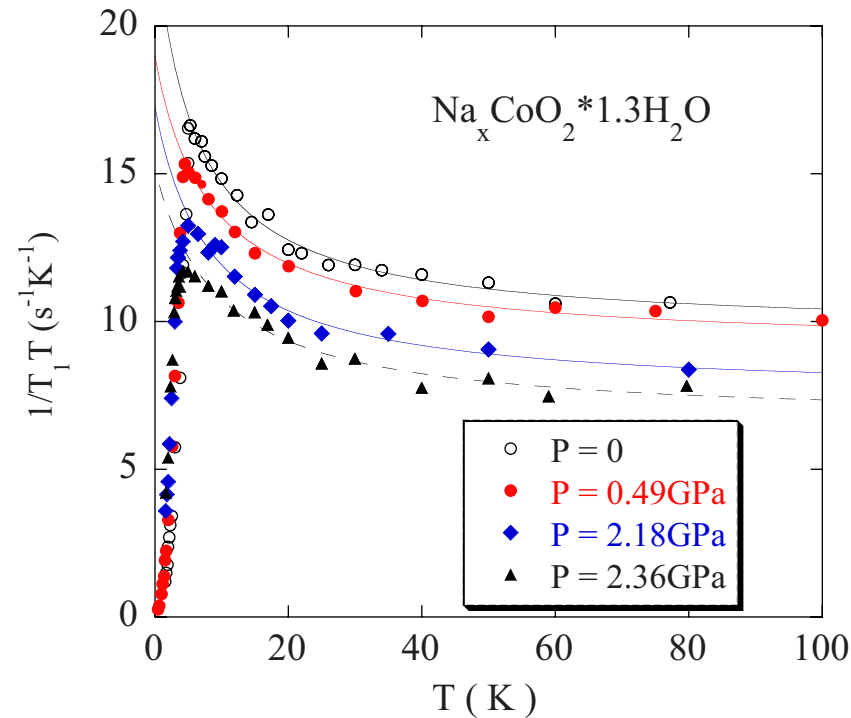

FIG. 3. (Color online) $1 / T_{1} T$ as a function of temperature at ambient and high pressures. The curves are fits to $1 / T_{1} T$ $=\left(1 / T_{1} T\right)_{0}+\left(80 \mathrm{~s}^{-1}\right) /(T+\theta)$. The resulting $\left(1 / T_{1} T\right)_{0}$ and $\theta$ are plotted in Fig. 4.

dynamical susceptibility. If one assumes that there is a peak around the AF wave vector $Q$, then one may have the following approximation:

$$
1 / T_{1} T=\left(1 / T_{1} T\right)_{A F}+\left(1 / T_{1} T\right)_{0}
$$

where $\left(1 / T_{1} T\right)_{0}$ denotes the contribution from wave vectors other than $Q$, which usually is dominated by the uniform susceptibility $\chi_{0}$. For the AF contribution, we use the formula for a two-dimensional antiferromagnetically correlated metal, namely, $\left(1 / T_{1} T\right)_{A F}=C /(T+\theta) .{ }^{13}$ We find that this model fits the data quite well, as depicted by the curves in Fig. 3. The resulting fitting parameters are shown in Fig. 4. Note that a small value of $\theta$ means that the system is close to the AF instability $(\theta=0)$. Therefore, the increase of $\theta$ to a larger positive value indicates a weakening of the antiferromagnetic correlations. On the other hand, the decrease of $\left(1 / T_{1} T\right)_{0}$ can be attributed to the decrease of the density of states at the Fermi level, $N\left(E_{F}\right)$, since $\left(1 / T_{1} T\right)_{0} \propto N\left(E_{F}\right)^{2}$.

As seen in Fig. 4, overall, $T_{c}$ decreases with increasing pressure, but nonmonotonically. The average rate of $d T_{c} / d P$ in the whole $P$ range is $-0.49 \pm 0.09 \mathrm{~K} / \mathrm{GPa}$. The average rate below $1.6 \mathrm{GPa}$ is $-0.28 \pm 0.05 \mathrm{~K} \mathrm{GPa}$, which is in good agreement with the rate $-0.27 \pm 0.03 \mathrm{~K} / \mathrm{GPa}$ reported by Lorenz et al., ${ }^{8}$ who measured the ac susceptibility in this pressure range. However, in the measurements of Lorenz et al., $T_{c}$ is $P$ insensitive in the range of $P \leqslant 0.5 \mathrm{GPa}$, while in the present study, $T_{c}$ is $P$ insensitive in the range of $0.49 \leqslant P \leqslant 1.93 \mathrm{GPa}$. The reason for the discrepancy is unknown at this point. It is noted that $\nu_{Q}$ increases smoothly in the whole pressure range, which assures that the pressure is indeed transmitted to the sample.

Below $0.49 \mathrm{GPa}, \theta$ increases and $\left(1 / T_{1} T\right)_{0}$ decreases, which indicates that the decrease of $T_{c}$ is accompanied by both weakening of the spin correlations and reduction of the DOS. The pressure effects on $T_{c}, \theta$, the DOS, and $\nu_{Q}$ are 


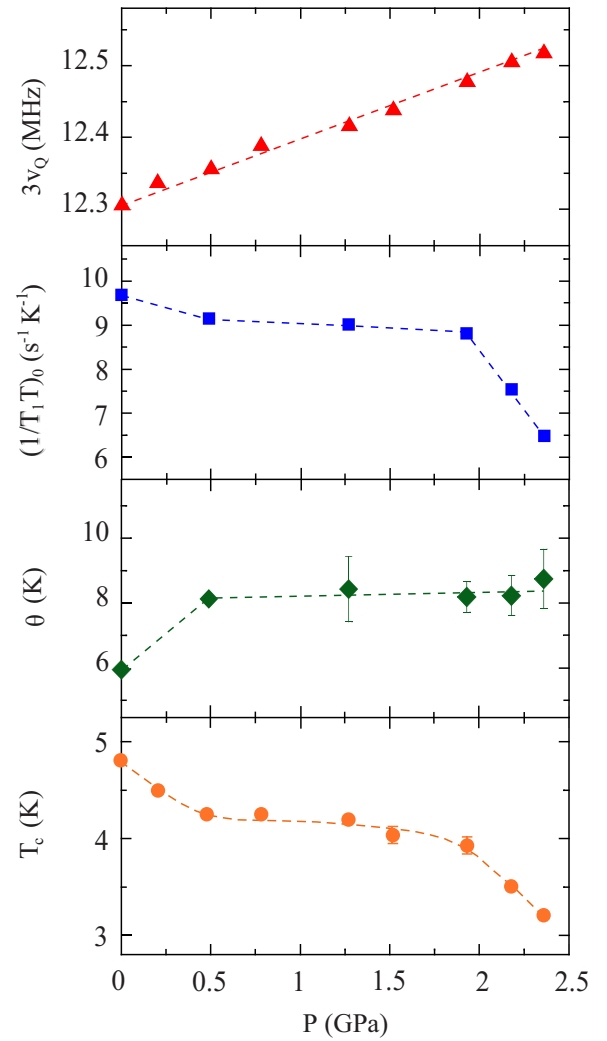

FIG. 4. (Color online) Various parameters obtained in this study plotted as a function of pressure. The dotted curves are guides to the eyes. The error in estimating the peak frequency $3 \nu_{Q}$ and $\left(1 / T_{1} T\right)_{0}$ is within the size of the symbols. Note that the density of states is proportional to $\sqrt{\left(1 / T_{1} T\right)_{0}}$.

equivalent to increasing the $\mathrm{Na}$ content. ${ }^{5}$ This suggests that the pressure effect in this range is to induce a charge redistribution between Co and oxygen. On the other hand, at pressures above $1.93 \mathrm{GPa}$, the DOS decreases with increasing $P$, while $\theta$ is less $P$ dependent, suggesting that the reduction of $T_{c}$ at high pressures is primarily due to the reduction of the DOS. Although several mechanisms for the superconductivity, ${ }^{14-19}$ including electron-lattice interaction, ${ }^{14}$ and a charge-order-driven mechanism, ${ }^{15}$ have been proposed, our result indicates that the Co electronic state (namely, the DOS) and magnetic fluctuations of likely antiferromagnetic origin are primarily responsible for the superconductivity.

Finally, we turn to the superconducting state. Figure 5 shows the temperature dependence of $1 / T_{1}$ at $P=0$ and $0.49 \mathrm{GPa}$. It can be seen that $1 / T_{1}$ follows a $T^{3}$ variation down to the lowest temperature measured $\left(\sim 0.12 T_{c}\right)$. This result establishes that the sample is free of impurity scattering, and indicates unambiguously that line nodes exist in the gap function. As we noted in previous publications, ${ }^{2,5}$ the gap function with line nodes generates an energy- $(E-)$ linear DOS at low $E$, which results in a $T^{n}(n=3)$ dependence of $1 / T_{1}$. Low- $E$ excitations arising from impurity scattering or other competing orders usually modify the DOS near the Fermi level, leading to an exponent $n$ less than 3. Also, an

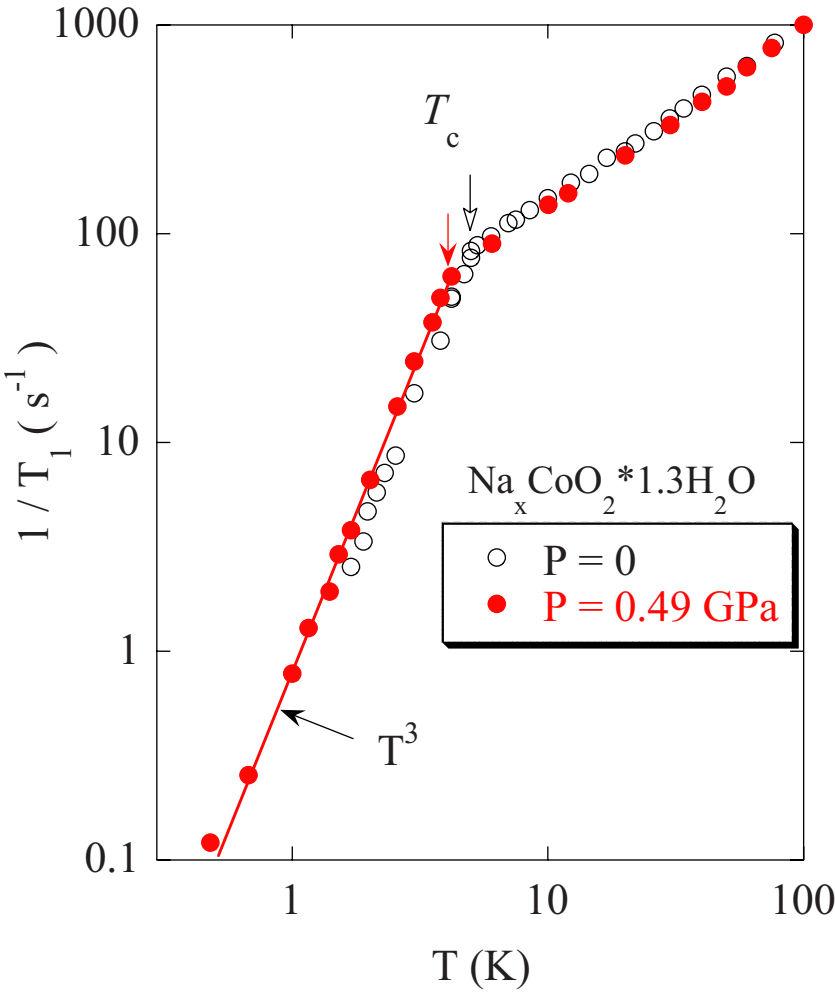

FIG. 5. (Color online) Temperature dependence of $1 / T_{1}$ at ambient pressure and $P=0.49 \mathrm{GPa}$. The arrow indicates $T_{c}$ at different pressures. The straight line depicts the $T^{3}$ variation.

anisotropic $s$-wave gap would give rise to a coherence peak just below $T_{c}$, which is incompatible with the experimental result. It is noted that the existence of nodes is consistent with superconductivity being induced by electron correlations.

In conclusion, we have presented systematic ${ }^{59} \mathrm{Co} \mathrm{NQR}$ measurements on the cobalt oxide superconductor $\mathrm{Na}_{x} \mathrm{CoO}_{2} \cdot 1.3 \mathrm{H}_{2} \mathrm{O}$ under high pressures up to $2.36 \mathrm{GPa}$. We find that $T_{c}$ decreases with increasing pressure at an average rate of $-0.49 \pm 0.09 \mathrm{~K} / \mathrm{GPa}$. At low pressures $P \leqslant 0.49 \mathrm{GPa}$, the decrease of $T_{c}$ is accompanied by both a weakening of the spin correlations at a finite wave vector and a reduction of the density of states at the Fermi level. At high pressures above $1.93 \mathrm{GPa}$, however, the decrease of $T_{c}$ is due mainly to a reduction of the density of states. These results indicate that the electronic and magnetic state of Co is primarily responsible for the superconductivity; namely, both a large DOS and spin correlations are indispensable for a high $T_{c}$ in this class of materials. The spin-lattice relaxation rate $1 / T_{1}$ at $P=0.49 \mathrm{GPa}$ shows a $T^{3}$ variation below $T_{c}$ down to $T \sim 0.12 T_{c}$, which provides compelling evidence for the presence of line nodes in the superconducting gap function.

We thank M. Nishiyama and K. Katayama for assitance in some of the measurements. This work was supported in part by research grants from MEXT, NSF, the T. L. L. Temple Foundation, the John and Rebecca Moores Endowment, and DOE. 
${ }^{1}$ K. Takada, H. Sakurai, E. Takayama-Muromachi, F. Izumi, R. A. Dilanian, and T. Sasaki, Nature (London) 422, 53 (2003).

${ }^{2}$ T. Fujimoto, G.-Q. Zheng, Y. Kitaoka, R. L. Meng, J. Cmaidalka, and C. W. Chu, Phys. Rev. Lett. 92, 047004 (2004).

${ }^{3}$ Y. Bang, M. J. Graf, and A. V. Balatsky, Phys. Rev. B 68, 212504 (2003).

${ }^{4}$ Q. Han and Z. D. Wang, Phys. Rev. B 70, 184504 (2004).

${ }^{5}$ G.-Q. Zheng, K. Matano, R. L. Meng, J. Cmaidalka, and C. W. Chu, J. Phys.: Condens. Matter 18, L63 (2006).

${ }^{6}$ Y. Kobayashi, M. Yokoi, and M. Sato, J. Phys. Soc. Jpn. 72, 2453 (2003).

${ }^{7}$ G.-Q. Zheng, K. Matano, D. P. Chen, and C. T. Lin, Phys. Rev. B 73, 180503(R) (2006).

${ }^{8}$ B. Lorenz, J. Cmaidalka, R. L. Meng, and C. W. Chu, Phys. Rev. B 68, 132504 (2003).

${ }^{9}$ J. Chepin and J. H. Ross, J. Phys.: Condens. Matter 3, 8103 (1991).
${ }^{10}$ K. Murata, H. Yoshino, H. O. Yadav, Y. Honda, and N. Shirakawa, Rev. Sci. Instrum. 68, 2490 (1997).

${ }^{11}$ T. F. Smith, C. W. Chu, and M. B. Maple, Cryogenics 9, 53 (1969).

${ }^{12}$ D. J. Singh, Phys. Rev. B 61, 13397 (2000).

${ }^{13}$ T. Moriya, K. Ueda, and Y. Takahashi, J. Phys. Soc. Jpn. 59, 2905 (1990).

${ }^{14}$ K. Yada and H. Kontani, J. Phys. Soc. Jpn. 75, 033705 (2006).

${ }^{15}$ O. I. Motrunich and P. A. Lee, Phys. Rev. B 70, 024514 (2004).

${ }^{16}$ M. D. Johannes, I. I. Mazin, D. J. Singh, and D. A. Papaconstantopoulos, Phys. Rev. Lett. 93, 097005 (2004).

${ }^{17}$ B. Liu, Y. Liang, and S. Feng, Commun. Theor. Phys. 43, 1127 (2005).

${ }^{18}$ K. Kuroki, S. Ohkubo, T. Nojima, R. Arita, S. Onari, and Y. Tanaka, Phys. Rev. Lett. 98, 136401 (2007).

${ }^{19}$ M. Ogata, J. Phys. Soc. Jpn. 72, 1839 (2003). 